\title{
Alterations in functional connectivity are associated with white matter lesions and information processing efficiency in multiple sclerosis
}

\author{
José Miguel Soares ${ }^{1,2} \cdot$ Raquel Conde $^{3} \cdot$ Ricardo Magalhães $^{1,2,4} \cdot$ Paulo Marques $^{1,2} \cdot$ Rosana Magalhães $^{5}$. \\ Luciana Gomes $^{5}$ • Óscar F. Gonçalves ${ }^{5,6,7}$ • Mavilde Arantes ${ }^{3,8}$ • Adriana Sampaio ${ }^{5}$
}

Published online: 29 February 2020

(C) Springer Science+Business Media, LLC, part of Springer Nature 2020

\begin{abstract}
Functional connectivity (FC) is typically altered in individuals with Multiple Sclerosis (MS). However, in relapsing-remitting multiple sclerosis (RRMS) patients, the relationship between brain FC, tissue integrity and cognitive impairment is still unclear as contradictory findings have been documented. In this exploratory study we compared both the whole brain connectome and resting state networks (RSNs) FC of twenty-one RRMS and seventeen healthy controls (HCs), using combined network based statistics and independent component analyses. The total white matter (WM) lesion volume and information processing efficiency were also correlated with FC in the RRMS group. Both whole brain connectome and individual RSNs FC were diminished in patients with RRMS compared to HC. Additionally, the reduction in FC was found to be a function of the total WM lesion volume, with greatest impact in those harboring the largest lesion volume. Finally, a positive correlation between FC and information processing efficiency was observed in RRMS. This complimentary whole brain and RSNs FC approach can contribute to clarify literature inconsistencies regarding FC alterations and provide new insights on the white matter structural damage in explaining functional abnormalities in RRMS.
\end{abstract}

Keywords Multiple sclerosis · White matter lesion · Resting State $\cdot$ Functional connectivity $\cdot$ Information processing efficiency

\section{Introduction}

Multiple Sclerosis (MS) is a demyelinating and progressive disorder of the central nervous system that is often (40-65\%) accompanied by cognitive impairment. This is

José Miguel Soares and Raquel Conde contributed equally to this work.

Electronic supplementary material The online version of this article (https://doi.org/10.1007/s11682-020-00264-z) contains supplementary material, which is available to authorized users.

José Miguel Soares

josesoares@med.uminho.pt

1 Life and Health Sciences Research Institute (ICVS), School of Medicine, University of Minho, Campus Gualtar, 4710-057 Braga, Portugal

2 ICVS/3B's, PT Government Associate Laboratory, Braga/ Guimarães, Portugal

3 University of Porto, Faculty of Medicine, Porto, Portugal

4 Clinical Academic Center-Braga, Braga, Portugal manifested in such functions as memory and informationprocessing speed, as well as in motor and sensory deficits (Jongen et al. 2012), being depression and fatigue common among MS patients (Braley and Chervin 2010; Skokou et al. 2012).
5 Psychological Neuroscience Lab, CIPsi, School of Psychology, University of Minho, Braga, Portugal

6 Social and Cognitive Neuroscience Laboratory and Developmental Disorders Program, Center for Health and Biological Sciences, Mackenzie Presbyterian University, Sao Paulo 01241-001, Brazil

7 Spaulding Center of Neuromodulation, Department of Physical Medicine \& Rehabilitation, Spaulding Rehabilitation Hospital and Massachusetts General Hospital, Harvard Medical School, Boston, MA, USA

8 Division of Neuroradiology, Department of Radiology, Portuguese Institute of Oncology, Porto, Portugal 
Impairment is thought to emerge from compromised neuronal connectivity as a consequence of multiple mechanisms such as macroscopic lesions of white matter (WM) (Chiaravalloti and DeLuca 2008; Massimo Filippi et al. 2018), diffuse microscopic WM abnormalities (De Santis et al. 2019) as well as cortical lesions (Calabrese et al. 2015; Emanuele et al. 2017). Anatomical Magnetic Resonance Imaging (MRI) is a key component of MS diagnosis (Polman et al. 2011) and also has led to improved understanding of its pathophysiology (Castellazzi et al. 2018; M. Filippi et al. 2011; Magliozzi et al. 2018; Tewarie et al. 2018a). More recently, functional MRI (fMRI) has emerged as a fundamental technique used to assess the impact of brain alterations in the cortico-cortical and cortico-subcortical connections by looking at brain functional connectivity (FC), both at the whole brain connectome and within specific networks known as the Resting State Networks (RSNs) (Fox and Raichle 2007; Jaeger et al. 2018; Marques et al. 2016; Soares et al. 2016).

Alterations of FC have been demonstrated in a number of neurological and neuropsychiatric disorders (Deco and Kringelbach 2014; van den Heuvel and Hulshoff Pol 2010; Zhang and Raichle 2010) including MS (Rocca et al. 2010; Sbardella et al. 2015a). Overall, whereas increased FC has been described in early MS (Louapre et al. 2014) and clinically isolated syndrome (Yaou Liu et al. 2016; Roosendaal et al. 2010), studies in patients with chronic secondary progressive and primary progressive MS have shown a decreased FC with disease progression (Rocca et al. 2010). Nevertheless, results have been inconsistent in relapsing-remitting MS (RRMS) subjects, where both FC increases and decreases have been reported (Sbardella et al. 2015b; Tahedl et al. 2018). Regarding specific networks, RRMS patients have shown abnormalities in the default mode network (DMN) FC especially in cognitively impaired patients (Louapre et al. 2014) and reduced $\mathrm{FC}$ in the DMN, sensorimotor, cognitive, thalamic, attention and cerebellar networks, while simultaneously observing increased RS FC within visual/sensory and subcortical networks (Rocca et al. 2017; Roosendaal et al. 2010); furthermore, a decreased FC of the anterior cingulate cortex was also related with the level of cognitive impairment (Bonavita et al. 2011; Rocca et al. 2010). At the whole brain connectome, patients display a connectivity disruption accompanied by loss of network efficiency with impact on cognition (Fleischer et al. 2019; He et al. 2009; Y. Liu et al. 2018; Rocca et al. 2016).

These inconsistencies among studies derive from different factors, including disease duration, topology of the lesion and lesion load, among others (Castellazzi et al. 2018; Tewarie et al. 2018b). In fact, structural network disconnection has been previously associated with increased FC (Patel et al. 2018), with others suggesting that white matter damage has an inverted U-curve effect on functional connectivity, with an initial increase followed by a decrease in overall FC (Tewarie et al. 2018a). While it is evident that FC in MS patients is altered, the precise relationship between structural lesion and complex functional networks is also not clear. Both gray and WM lesions have impact on clinical symptoms and cognition, but the separate effects of gray matter and WM structural changes in MS and its relation with functional connectivity findings are scarce. Therefore, there is a compelling need to delineate how changes in the physical integrity of brain tissue correlate with modified FC (Droby et al. 2016; He et al. 2009; Langen et al. 2017; Tewarie et al. 2018b).

Abnormalities of FC are of significant clinical relevance, as they have regularly been associated with cognitive impairment and physical disability (Hawellek et al. 2011; Rocca et al. 2010; Rocca et al. 2016). However, the heterogeneity of FC findings in RRMS, potentially due to the wide spectrum of clinical manifestations of the MS clinical phenotype as well as the different methodologies employed for functional analyses, makes it difficult to understand the link between structural damage, network collapses and cognitive decline. Struck by the network FC discrepancies and the unclear link with structural damage and cognition in MS, in this exploratory study, we have assessed for the first time, combined and complimentary whole brain connectome and specific within RSNs FC, in the same study and cohort of RRMS subjects when compared to Healthy Controls (HC). Additionally, we have explored the crucial association with total WM lesion volume and information processing efficiency, while controlling for depression status. We hypothesized that, independently of the FC methodologic approach, RRMS patients would present an altered FC when compared to $\mathrm{HC}$, with FC being negatively associated with the structural lesioning and cognitive status.

\section{Materials and methods}

\section{Participants and neuropsychological assessment}

The study included a group of 21 participants with clinically definitive RRMS, recruited from the database of the Neurology Department of a General Hospital [Hospital Geral de Coimbra (Covões) - Centro Hospitalar de Coimbra] and referred by the neurologists associated with the project.

The following patient eligibility criteria was employed (a) definite MS diagnosis (Polman et al. 2011); (b) RRMS course of disease; (c) stable disease on the 3 months previous to the study (no attacks); (d) neurological disability level Expanded Disability Status Scale (EDSS) less than 5; (e) treatment with imunomodulatory medication; and (f) right handedness. Exclusion criteria for both groups included: (a) the presence of any medical condition that could influence cognition; (b) history of brain injury; (c) psychiatric disorder (current or past); (d) psychoactive substances abuse (current or past); (e) 
severe visual impairments; and (f) incapacity and/or inability to attend the MRI session.

The mean disability level as assessed by the EDSS had a median of 1.5 (ranging from 0.0 to 5.0). Mean time from diagnosis was $17.7( \pm 14.51)$ months, ranging from 1 to 48 months. A HC group included 17 healthy adults recruited from the community with similar distribution of age and gender proportion.

The neuropsychological protocol included the Minimal Assessment of Cognitive Function in MS (MACFIMS) (Benedict et al. 2002), which is a battery based on a consensus of experts in the field that covers the assessment of the cognitive domains typically affected in MS, namely processing speed, memory (particularly working memory), executive functioning and verbal fluency. For the purpose of this study we reported only two tests (Symbol Digit Modalities Test SDMT and Paced Auditory Serial Addition Test - PASAT) which have been proven to be sensitive to subtle deficits in MS, show good psychometric properties and have been associated with neuroimaging findings (Cardinal et al. 2008; Christodoulou et al. 2003). It has been suggested that processing speed deficit is the core cognitive deficit of MS and the SDMT is the best available measure to detect it (Benedict et al. 2006; Benedict et al. 2012; DeLuca et al. 2004), being an useful screening measure for brain damage and cerebral dysfunction (Strauss et al. 2007). To monitor the cognitive impairment, the PASAT was also applied (Benedict et al. 2002). Considering these evidence and based on previous reports (Sumowski et al. 2009), we have created a composite of information processing efficiency that was calculated as the average of the z-scores in SDMT and PASAT. Mood was also assessed, using the standard Beck Depression Inventory (BDI) (Beck et al. 1961) Portuguese version from Vaz Serra and Pio Abreu (1973).

All participants were right-handed and group differences of descriptive statistics are presented in Table 1.

\section{Data acquisition}

Participants were scanned on a clinical approved Siemens Trio Tim 3.0 T (Siemens Medical Solutions, Erlangen, Germany) MRI. Imaging protocols included one structural T1 - weighted acquisition and one functional $\mathrm{T} 2 *$ - weighted acquisition, conducted in the same session. For structural analysis, a T1 high-resolution anatomical sequence, 3D MPRAGE (magnetization prepared rapid gradient echo) was performed with the following scan parameters: repetition time $(T R)=2.300 \mathrm{~s}$, echo time $(\mathrm{TE})=2.98 \mathrm{~ms}, 160$ sagittal slices with no gap, flip angle $(\mathrm{FA})=9^{\circ}$, Field of View $(\mathrm{FoV})=256 \mathrm{~mm}$, in-plane resolution $=1.0 \times 1.0 \mathrm{~mm}^{2}$ and slice thickness $=1.0 \mathrm{~mm}$. During the RS fMRI acquisition, using gradient echo weighted echoplanar images (EPIs), the participants were instructed to keep their eyes closed and to think about nothing in particular. The imaging parameters were: 150 volumes, $\mathrm{TR}=2.0 \mathrm{~s}, \mathrm{TE}=$ $30 \mathrm{~ms}, \mathrm{FA}=90^{\circ}$, in-plane resolution $=3.0 \times 3.0 \mathrm{~mm}^{2}, 46$ interleaved slices, slice thickness $=3 \mathrm{~mm}$ and FoV $=240 \mathrm{~mm}$.

\section{Image pre-processing}

RS fMRI data preprocessing was performed using the FMRIB Software Library (FSL v5.07; http://fsl.fmrib.ox.ac.uk/fsl/) tools. To achieve signal stabilization and allow participants to adjust to the scanner noise, the first 5 fMRI volumes $(10 \mathrm{~s})$ were discarded using fslroi. Images were firstly corrected for slice timing using the first slice as reference using slicetimer. To correct for head motion, images were realigned to the middle image with a six-parameter rigid-body spatial transformation using mcflirt. Images were then spatially normalized with a non-linear transformation to the MNI (Montreal Neurological Institute) standard coordinate system, being re-sampled to $2 \times 2 \times 2 \mathrm{~mm}^{3}$ (same resolution as the MNI template) using trilinear interpolation using flirt/fnirt and smoothed to decrease spatial noise with a $8 \mathrm{~mm}$, full width at half maximum (FWHM), Gaussian kernel using fslmaths. Resting state images were finally temporally band-pass filtered $(0.01-0.08 \mathrm{~Hz})$ using fslmaths. After pre-processing, two participants were excluded from the analyses steps as they presented head motion higher than $2 \mathrm{~mm}$ in translation and/or $1^{\circ}$ in rotation.

\section{Measurement of total WM lesion volume}

After the visual inspection of the 3D T1 MPRAGE images of each participant by one certified neuroradiologist (MA) and confirming that they were not affected by critical head motion, the total WM lesion volume was manually calculated. Lesions were firstly identified and manually outlined (based on the T1 hypointensities) in the three planes of the images (coronal, sagittal and axial) by a certified neuroradiologist (MA) and then checked by two observers (JMS and RC), blinded to patient's clinical and cognitive status. Each individual lesion's volume in $\mathrm{mm}^{3}$ was then summed to constitute the total WM lesion volume for each participant. The procedures of identification, segmentation and volumetric quantification of the lesions were performed using the 3D Slicer Software (htts:// www.slicer.org/).

\section{Network construction}

Testing of FC was performed both at the whole brain connectome level and within each RSN level.

A general linear model (usign $f s l \_g l m$ ) was used to remove the effect of several confounds. The mean WM and cerebrospinal fluid signals, 6 motion parameters and motion outliers (calculated using fsl_motion_outliers) were included. 
Table 1 Descriptive statistics of the study participants

\begin{tabular}{llll}
\hline Characteristics & MS & HC & Difference \\
\hline Males/Females & $9 / 12$ & $7 / 10$ & $\chi 2=0.01 ; p=0.917$ \\
Mean Age (Stdev); range & $37.05(9.29) ; 20-55$ & $35.87(8.01) ; 23-55$ & $t=0.389 ; p=0.6996$ \\
SDMT (Stdev); range & $46.95(8.35) ; 32-62$ & $61.93(12.15) ; 27-76$ & $t=-2.567 ; p=0.0146$ \\
PASAT (Stdev); range & $35.05(11.04) ; 14-55$ & $43.63(8.96) ; 22-57$ & $t=-1.727 ; p=0.0927$ \\
Information processing efficiency composite (Stdev); range & $-1.02(0.76) ;-2.38$ to 0.41 & $0.66(0.85) ;-2.18$ to 1.10 & $t=-3.81 ; p=0.0005$ \\
BDI (Stdev); range & $11.0(7.8) ; 0-28$ & $3.8(3.9) ; 0-12$ & $t=3.11 ; p=0.0036$ \\
\hline
\end{tabular}

For the whole-brain connectome analysis, mean time-series for 116 cortical, subcortical and cerebellar regions from the Anatomical Automatic Labeling (AAL) (Tzourio-Mazoyer et al. 2002) atlas were extracted. A symmetric adjacency matrix $\mathrm{R}$ was then produced, where each cell $\mathrm{r}_{i j}$ corresponded to the correlation coefficient (r) between the time-series of regions $i$ and $j$. This matrix was then transformed with Fisher's r-to-Z transformation to convert Pearson correlation coefficients between the 116 time-series to normally distributed Zvalues. The network node connections can be classified in short-range connections (interactions between anatomically adjacent nodes) and long-range connections (projections from distant anatomical areas/nodes), based on the Euclidean distance between the center of gravity of the nodes.

Group Independent Component Analysis (gICA) was performed with the entire sample (HC and RRMS groups) using FSL MELODIC (Multivariate Exploratory Linear Optimized Decomposition into Independent Components) (Beckmann and Smith 2004). This procedure involves the temporal concatenation of each subject's dataset into a single 4D dataset in order to perform group-wise ICA. The number of components to extract was automatically estimated by the software.

Group RSNs were visually identified, including the DMN, attention networks (dorsal attention network - DAN and ventral attention network - VAN), sensorimotor, salience, basal ganglia, precuneus, visual (primary and higher), auditory and language. In order to extract the subject's specific components corresponding to the group-wise identified RSNs of interest, the dual regression procedure using randomise was employed. The dual regression procedures work in two stages for each subject: first the group averaged spatial maps are (through a multiple regression) regressed into the subject 4D space dataset, creating a unique time-series for each RSN. Next, the time-series are regressed into the subject's 4D dataset, resulting in a spatial map for each subject and for each network chosen. Mean WM and cerebrospinal fluid signals, and the six motion parameters resulting from the motion correction step were included in the matrix for the second step of the dual regression in order to correct for its influence in the subject specific spatial maps. The group spatial map of each network was then thresholded at its 99th percentile in order to extract the clusters that define each RSN.

\section{Statistical analyses}

Characterization of the samples was done using the IBM SPSS Statistics software, v.22 (IBM, New York) and Matlab R2016b. Comparisons between groups were performed with two-tailed independent-samples t-test and a chi square test for the gender. For all these comparisons, the significance level was set at 0.05 . Values are presented as mean \pm standard deviation of the mean on Table 1.

Whole brain connectivity networks were analyzed through a Network Based Statistics (NBS) approach (Zalesky et al. 2010). NBS procedure consists first in the application of a statistical model independently for each individual network connection and thresholding these by a user-defined threshold. Thereafter, the identification of sub-networks composed of connections that survive the primary threshold is performed, and its significance according to the network size is determined. The sub-networks significance is calculated by comparing their sizes with the distribution of the subnetworks size obtained through 5000 random permutations of the original hypothesis. Because different user defined thresholds can yield topologically different networks, three standard different connection thresholds were tested at $p<0.01,0.005$ and 0.001 . Results were considered significant at $p<0.05$ corrected for multiple comparisons at the network level. Similarly to the local connectivity, independent two samples t-tests were used to test for group differences and two multiple regressions to explore the correlation between whole brain functional connectivity with the total WM lesion volume and the composite of information processing efficiency.

RSNs FC analyses were performed using the second-level random effect analyses in SPM12 (http://www.fil.ion.ucl.ac. $\mathrm{uk} / \mathrm{spm} / \mathrm{software} / \mathrm{spm} 12 /$ ). Initially, analyses were performed only to confirm each RSN FC, using one-sample t-tests for each group and each RSN in study. Thereafter, two-sample ttests were performed to compare each RSN between the RRMS and HC groups. Finally, to investigate the impact of the total WM lesion volume and the information processing efficiency composite on the FC, two multiple regression analysis were performed between each RSN FC and the total WM lesion volume (normalized for each participant's total 
intracranial volume) and the information processing efficiency composite, controlling for BDI. We controlled for depression symptoms considering its high prevalence in MS as well as its high impact and relation with fatigue and cognitive alterations (Patrick et al. 2009; Skokou et al. 2012). All RSNs results were considered significant at $p<0.05$ corrected for multiple comparisons using the Monte Carlo correction based on the combination of a $p$ value and a minimum cluster size calculated for each RSN. A minimum cluster size of 54 voxels for the DMN, 11 for the DAN, 34 for the VAN, 32 for the sensorimotor, 35 for the salience, 18 for the basal ganglia, 30 for the precuneus, 23 for the primary visual, 28 for the higher visual, 20 for the auditory and 32 for the language network, calculated with AFNI's 3dClustSim, was used. Results were inclusively masked with the RSN templates from (Shirer et al. 2012).

Anatomical labelling for all results was defined by a combination of visual inspection and Anatomical Automatic Labelling atlas (AAL) (Tzourio-Mazoyer et al. 2002).

\section{Results}

\section{Participants description}

Descriptive statistics of the participants are presented in Table 1. Groups did not differ significantly in terms of age, gender and PASAT. On the other hand, groups differed in SDMT and information processing efficiency composite, with participants with RRMS presenting lower Z-Scores (worse performance). Mean total WM lesion volume in the RRMS was $2194,3 \mathrm{~mm}^{3}$ (STDev 1326,7 $\mathrm{mm}^{3}$ ), ranging from $637 \mathrm{~mm}^{3}$ to $4703 \mathrm{~mm}^{3}$.

\section{Whole brain Connectome}

Whole brain decreased network FC in the RRMS group was observed when compared to the $\mathrm{HC}$ for each of the three defined thresholds. In contrast, no network nodes or connections presented increased FC in RRMS for any threshold. The FC of both short and long-range connections was decreased in the RRMS group, as compared to HC. Based on the most significant threshold, the most evident $\mathrm{FC}$ decreases in the RRMS group were in the connections between frontal regions (the bilateral superior frontal gyri and the right middle frontal gyrus), the bilateral supramarginal gyrus, subcortical regions (the right amygdala, middle and posterior cingulate, insula and thalamus), temporal regions (the right Heschl gyrus, and the bilateral temporal superior regions) and the cerebellum. The significant regions for each threshold are presented in Table S1, and the relevant nodes and connections may be seen in Fig. 1A.

\section{Resting State network connectivity}

The FC within typical brain regions underlying the auditory, basal ganglia, DAN, DMN, high visual, language, precuneus, primary visual, salience, sensorimotor and VAN networks was initially confirmed for both groups (results not shown) in the one-sample t-test analyses. During RS, participants with RRMS were characterized by decreased FC in the auditory, DMN, primary visual, salience, sensorimotor and VAN networks and an increased FC in the DAN, when compared to controls. No differences were observed between RRMS and $\mathrm{HC}$ for the basal ganglia, high visual, language and precuneus networks. Significant regions within each RSN are displayed in Fig. 1B and described in Table 2.

\section{Association between Total WM lesion volume and RRMS functional connectivity}

After exploring the principal FC differences between RRMS and $\mathrm{HC}$, we investigated the influence of the total WM lesion volume on FC. A negative correlation between the total WM lesion volume and whole brain connectome's FC was found at all thresholds. No regions with positive correlation were found at any threshold. The FC of both short and long-range connections was affected by increased total WM lesion volume. Specifically, a subnetwork composed of nodes connecting the basal ganglia (the bilateral putamen and pallidum) as the central nodes, with the left thalamus, insula and posterior cingulate, the left superior frontal gyrus and the cerebellum, was characterized by a greater FC decrease in the presence of greater total WM lesion volume. The specific significant regions associated with the total WM lesion volume for each threshold are presented in Table S2 as the relevant nodes and connections in Fig. 2A.

At the RSN level, a significant negative correlation between the total WM lesion volume and FC for RRMS was found in the basal ganglia network, DAN, DMN, high visual, precuneus, salience, sensorimotor and VAN networks. No significant correlations were found with the auditory, language and primary visual networks. The significant regions within each RSN which were correlated with total WM lesion volume are displayed in Fig. 2B and detailed in Table 3.

\section{Association between information processing efficiency and RRMS functional connectivity}

At the connectome level, we observed a positive correlation between information processing efficiency composite and FC in the RRMS group for all the thresholds. Long-range connections between the frontal and cerebellar regions were the most predominant at the most significant threshold, i.e. a subnetwork composed of nodes connecting the middle and left superior orbitofrontal cortex, anterior cingulate cortex, left 
a
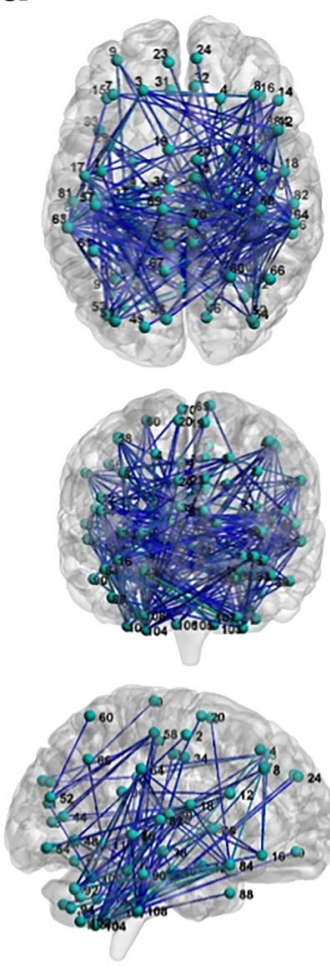

b

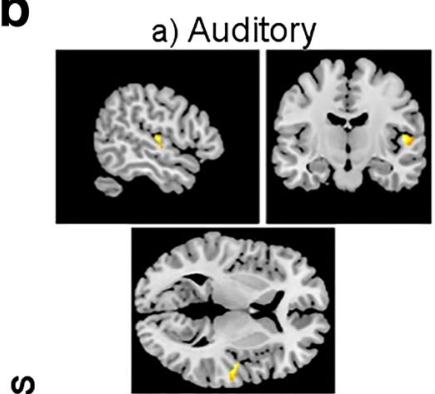

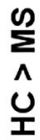

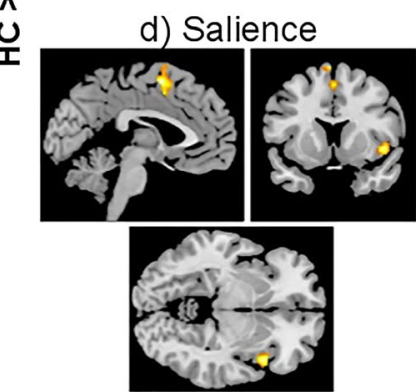

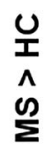

$p<0.005$
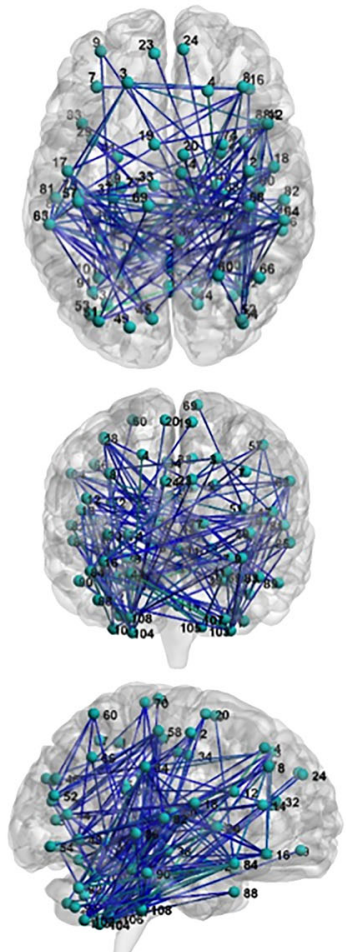

$p<0.001$
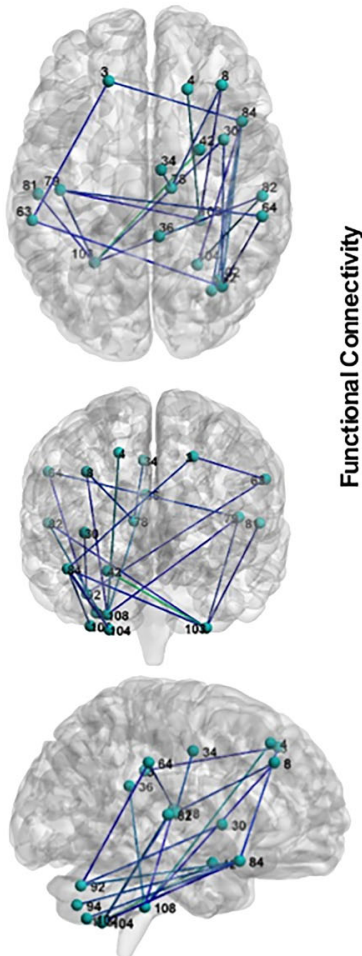

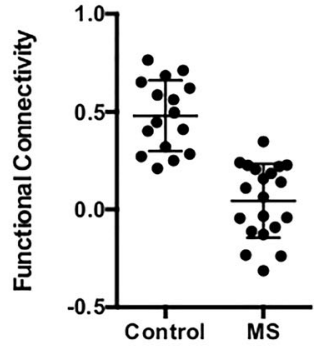

t-value

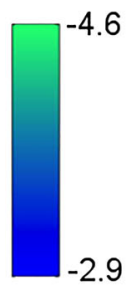

b) DMN

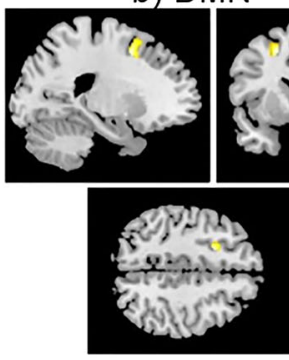

e) SMN

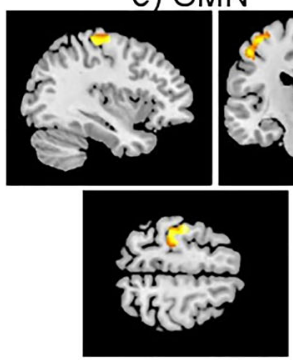

g) DAN

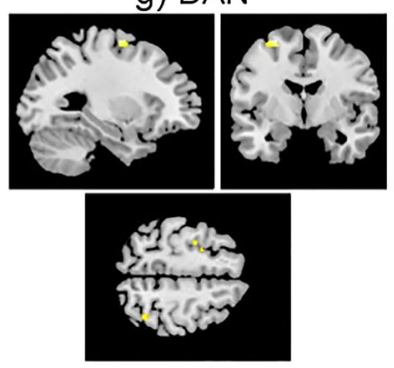

c) P Visual
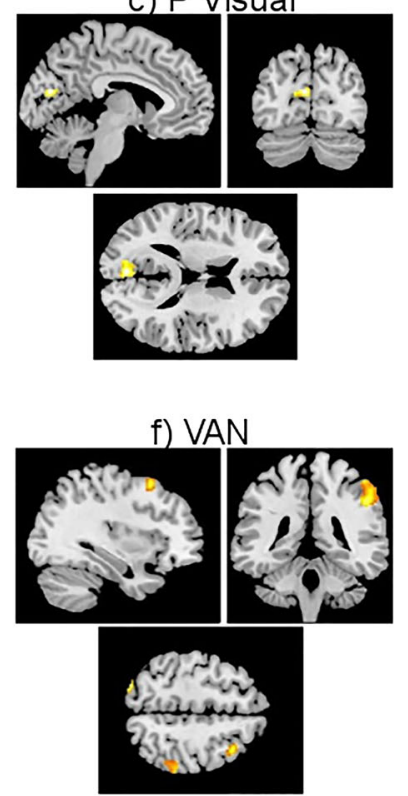

t-value

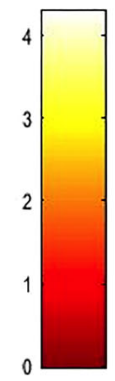


Fig. 1 Altered Functional Connectivity (FC) in Relapsing-Remitting Multiple Sclerosis (MS) patients compared to Healthy Controls (HC) at whole brain connectome (A - reduced FC in MS patients using primary thresholds of $\mathrm{p}<0.01, \mathrm{p}<0.005$ and $\mathrm{p}<0.001$, corrected for multiple comparisons) and within Resting State Networks (RSNs) (B, HC $>$ MS on top and $\mathrm{MS}>\mathrm{HC}$ on bottom, corrected for multiple comparisons)

caudate, right thalamus, cerebellum and vermis. The specific significant regions that correlated with the information processing efficiency for each threshold are presented in Table S3, with the relevant nodes and connections charted in Fig. 3A.

For specific RSNs, there was also a positive correlation between information processing efficiency and the FC of the DMN, precuneus, sensorimotor and VAN networks. No significant correlations were found with the auditory, basal ganglia, DAN, high visual, language, primary visual and salience networks. Specific significant regions within each RSN which correlated with information processing efficiency are displayed in Fig. 3B and described in Table 4.

\section{Discussion}

Alterations in the FC of individuals with MS have been well described (Rocca et al. 2010; Rocca et al. 2017; Sbardella et al. 2015a), however they are difficult to interpret based on the divergent results (both FC increases and decreases). As acknowledged by others, it is possible that the inconsistent findings, particularly evident in RRMS, result from differing methodological approaches, namely a more focused analysis on the individual RSNs FC (precluding complimentary whole brain FC information), differences in the disorder duration, clinical and cognitive spectrum phenotypes as well as variations in the degree of structural damage (Tewarie et al. 2018a). In this study, we dissected the FC differences between RRMS and $\mathrm{HC}$ not only at RSNs level, but also expanding it to a more holistic global network model of the entire brain using a connectome approach, while simultaneously exploring the influence of structural WM damage and the relation with an information processing efficiency index in the same cohort. The present data: i) confirmed the findings of FC decreases at the RSNs seen with RRMS, but also expands the observations to the whole brain connectome in the same cohort; ii) demonstrated the association between the total WM lesion volume load and the FC decrease in RRMS and; iii) found a positive correlation between brain FC and information processing efficiency in RRMS (independently of depressive symptoms).

\section{Whole brain connectome and resting state network's FC in RRMS}

At the whole brain level, a global widespread (cortical and subcortical) FC decrease was observed in the RRMS group as compared to $\mathrm{HC}$, which is consistent with other studies which showed a decrease in the FC of specific brain regions with disease progression (Bonavita et al. 2011; Rocca et al. 2010; Rocca et al. 2017) as well as findings consistent with a generalized network "collapse" (Schoonheim et al. 2015). While other studies which assessed structural network efficiency in MS concluded that the short-range connections

Table 2 Group differences in brain regions of the auditory, DMN, primary visual, salience, sensorimotor, VAN and DAN network maps (two sample ttests, corrected for multiple comparisons, $p<0.05$ )

\begin{tabular}{|c|c|c|c|c|c|}
\hline Condition & Network & Regions & Peak MNI coordinates & Cluster size & $\begin{array}{l}\text { Z- } \\
\text { Score }\end{array}$ \\
\hline \multirow[t]{13}{*}{$\mathrm{C}>\mathrm{MS}$} & Auditory & Right Heschl & $50,-12,6$ & 27 & 3.73 \\
\hline & \multirow[t]{2}{*}{$\mathrm{DMN}$} & Left superior frontal gyrus & $-22,8,48$ & 63 & 3.22 \\
\hline & & Left middle frontal gyrus & $-24,12,56$ & & 2.73 \\
\hline & Primary Visual & Left Calcarine & $-6,-76,16$ & 25 & 2.93 \\
\hline & \multirow[t]{2}{*}{ Salience } & Left Superior motor area & $-2,4,58$ & 60 & 3.79 \\
\hline & & Right Insula & $46,14,-4$ & 70 & 3.67 \\
\hline & \multirow[t]{2}{*}{ Sensorimotor } & Right Postcentral gyrus & $-48,-22,50$ & 243 & 3.73 \\
\hline & & Left Precentral gyrus & $-42,-14,60$ & & 3.61 \\
\hline & \multirow[t]{5}{*}{ VAN } & Right Supramarginal gyrus & $48,-38,40$ & 185 & 3.91 \\
\hline & & Right superior Parietal lobule & $48,-44,60$ & & 3.18 \\
\hline & & Right Angular gyrus & $52,-48,32$ & & 2.74 \\
\hline & & Right middle frontal gyrus & $36,16,56$ & 46 & 3.32 \\
\hline & & Left superior Parietal gyrus & $-24,-80,50$ & 31 & 3.19 \\
\hline \multirow[t]{2}{*}{$\mathrm{MS}>\mathrm{C}$} & \multirow[t]{2}{*}{ DAN } & Left superior Frontal gyrus & $-26,-4,64$ & 43 & 3.27 \\
\hline & & Left inferior Parietal gyrus & $-28,-44,42$ & 16 & 3.00 \\
\hline
\end{tabular}


a
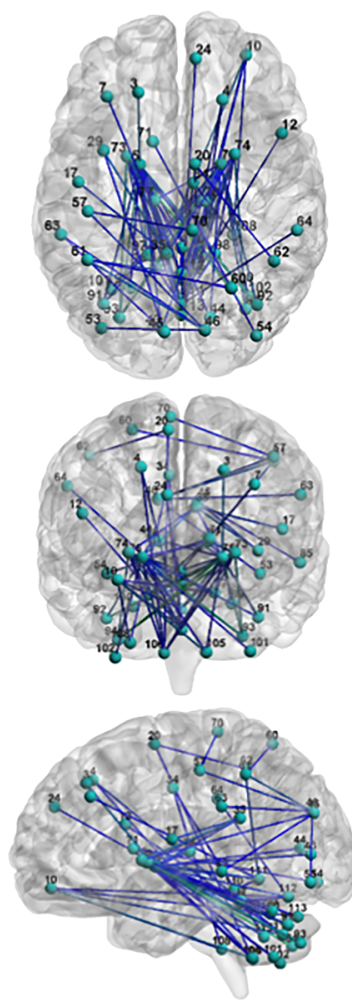

b
a) Basal Ganglia
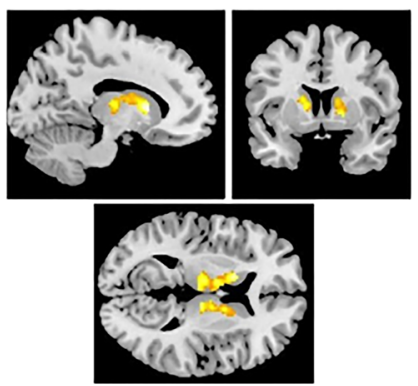

e) Precuneus
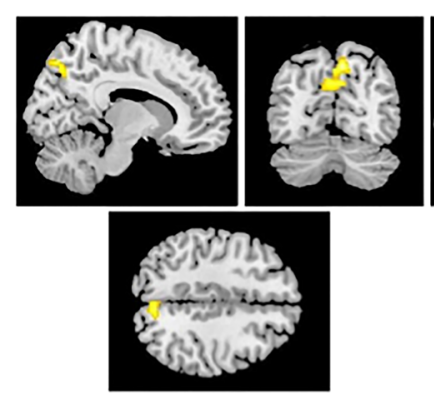

$p<0.005$
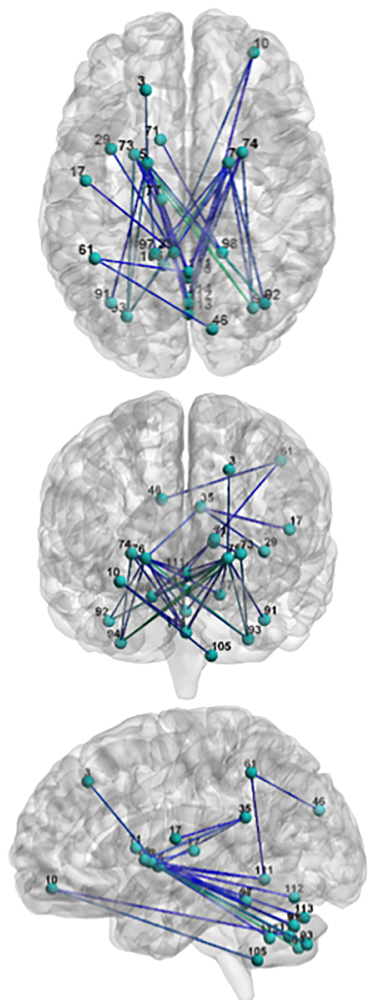

b) DAN

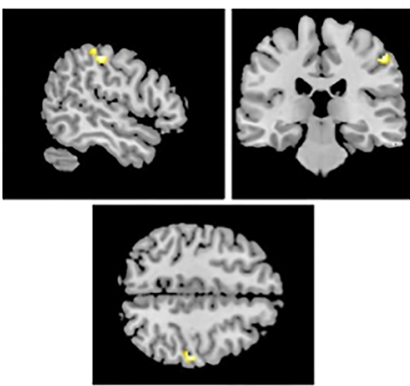

f) Salience
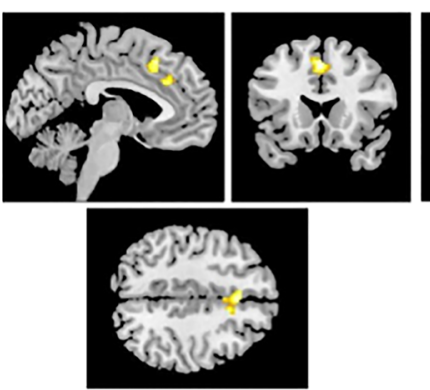

$\mathrm{p}<0.001$
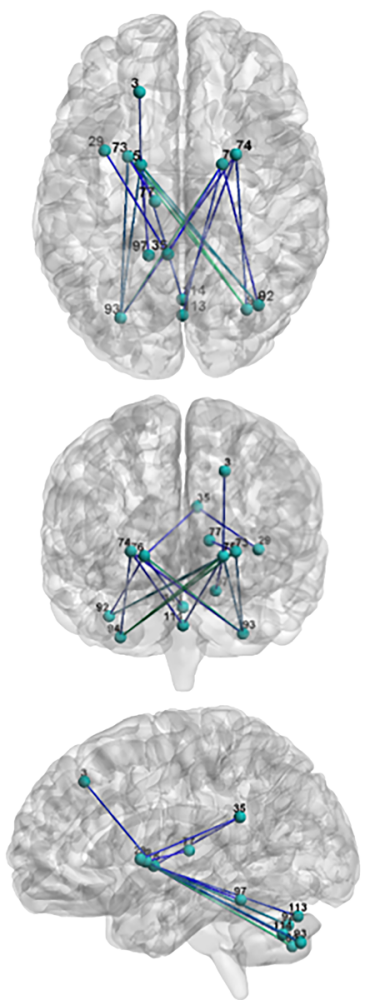

c) $\mathrm{DMN}$
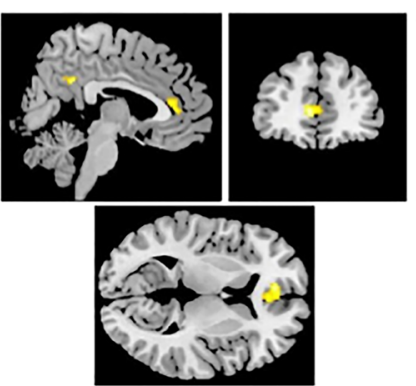

g) SMN
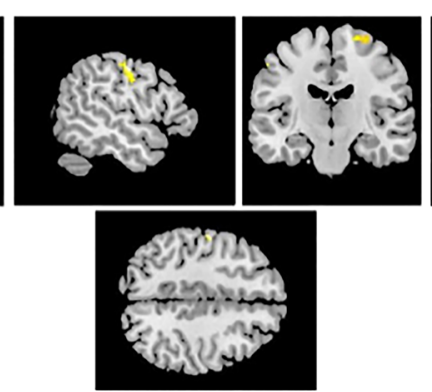

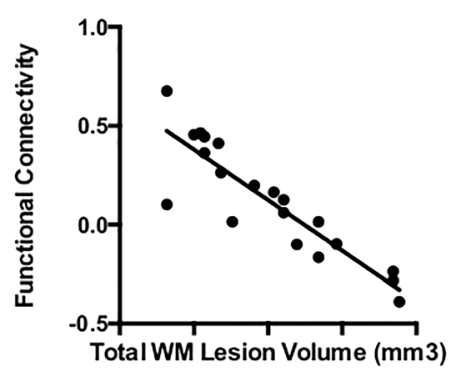

t-value

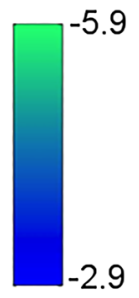

d) H Visual

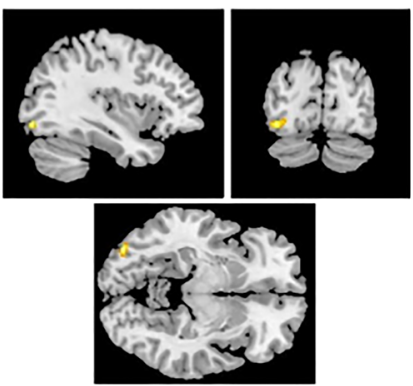

h) VAN
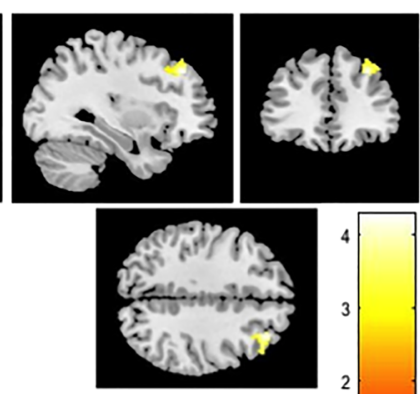

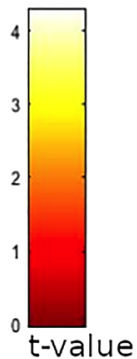


Fig. 2 Reduced Functional Connectivity (FC) in Relapsing-Remitting Multiple Sclerosis (MS) patients correlated with Total WM Lesion Volume at whole brain connectome (A, using primary thresholds of $p<0.01, p<0.005$ and $p<0.001$, corrected for multiple comparisons) and within Resting State Networks (RSNs, corrected for multiple comparisons) (B)

appear to be more impaired (He et al. 2009; Tahedl et al. 2018), our results suggest that at a functional level both short and long-connections seem to be equally affected. More specifically, a pronounced FC decrease was found in a subnetwork including: a) the superior frontal regions, consistent with previous findings showing a functional loss of "critical" frontal hubs in MS (Rocca et al. 2016); b) the insula, associated with decreased structural node efficiency in MS (He et al. 2009); c) the thalamus, thought to be involved in clinical manifestations of MS, such as fatigue, motor and cognitive deficits (Minagar et al. 2013; Rocca et al. 2016); d) the temporal pole, found to present a decreased structural efficiency in MS (He et al. 2009); and; e) the cerebellum, found to have a decreased connectivity in MS (Dogonowski et al. 2014; Rocca et al. 2017).

Our RSN results were characterized by the same pattern of FC decrease in all networks but the DAN. The FC decreases in RRMS, as compared to controls, in the auditory, primary visual and sensorimotor networks are likely to be related to the well-described motor and sensory deficits observed in many MS patients (Brownlee et al. 2017). Decreases in the FC of the DMN, but also herein extended to the salience and VAN networks, have also been described in MS and associated with cognitive impairment (Louapre et al. 2014; Rocca et al. 2010;
Rocca et al. 2017). Although we demonstrated FC decrease in local networks, increased FC of the DAN was observed. This result is consistent with other studies, suggesting that alterations in connectome and RSNs FC may be dissociated and a deeper understanding of other mechanisms that underlie FC alterations are still on debate (Dogonowski et al. 2014) and should consider brain disease duration, clinical and disability status as well as topology and structural damage (both gray and white matter).

\section{Association between WM lesioning and FC in RRMS}

Alterations in neural transmission along cortico-cortical and cortico-subcortical connections have been associated with MS disease-related WM lesions, and constitute functional evidence for the concept of a multisystem "disconnection syndrome" (He et al. 2009; Langen et al. 2017). Indeed, our data showed a direct correlation between the total WM lesion volume load and the reduction in the connectome's FC (both short and long-range connections equally affected), tending to support the described impact of the disease-related tissue damage upon brain FC. WM lesion load profoundly affected the FC connectome, more specifically a subnetwork including the superior frontal gyrus, insula, posterior cingulate cortex, putamen, pallidum, thalamus and the cerebellum. It has been postulated that regions such as the posterior cingulate cortex and the precuneus, known to present high metabolic rates at rest, are most vulnerable to the effects of disease and structural damage, as we have observed in our study (Cavanna and Trimble 2006; Rocca et al. 2017; Rocca et al. 2016). The FC

Table 3 Correlation between total WM lesion volume and RSNs FC in RRMS (multiple regressions, corrected for multiple comparisons, $\mathrm{p}<0.05$ )

\begin{tabular}{|c|c|c|c|c|c|}
\hline Correlation & Network & Regions & Peak MNI coordinates & Cluster size & $\begin{array}{l}\text { Z- } \\
\text { Score }\end{array}$ \\
\hline \multirow[t]{16}{*}{ Negative } & \multirow[t]{4}{*}{ Basal Ganglia } & Left Caudate & $-14,14,6$ & 186 & 3.43 \\
\hline & & Left Thalamus & $-12,-14,10$ & & 2.93 \\
\hline & & Right Thalamus & $10,-16,10$ & 40 & 3.08 \\
\hline & & Right Caudate & $12,4,12$ & 20 & 2.78 \\
\hline & DAN & Right Postcentral gyrus & $52,-26,48$ & 16 & 2.82 \\
\hline & \multirow[t]{3}{*}{ DMN } & Left Anterior cingulate cortex & $-8,46,6$ & 223 & 3.19 \\
\hline & & Right Posterior cingulate cortex & $12,-44,26$ & 60 & 3.05 \\
\hline & & Left Precuneus & $-2,-44,40$ & & 2.63 \\
\hline & High Visual & Left inferior Occipital gyrus & $-38,-82,-8$ & 32 & 3.39 \\
\hline & \multirow[t]{2}{*}{ Precuneus } & Right Precuneus & $6,-68,48$ & 174 & 2.90 \\
\hline & & Left Precuneus & $-4,-68,34$ & & 2.64 \\
\hline & \multirow[t]{2}{*}{ Salience } & Right Superior motor area & $4,14,58$ & 193 & 3.87 \\
\hline & & Right superior Frontal gyrus & $16,12,60$ & & 3.66 \\
\hline & \multirow[t]{2}{*}{ Sensorimotor } & Left Postcentral gyrus & $-54,-12,46$ & 40 & 3.07 \\
\hline & & Right Precentral gyrus & $28,-14,68$ & 34 & 2.49 \\
\hline & VAN & Right middle Frontal gyrus & $32,36,40$ & 99 & 2.64 \\
\hline
\end{tabular}


a
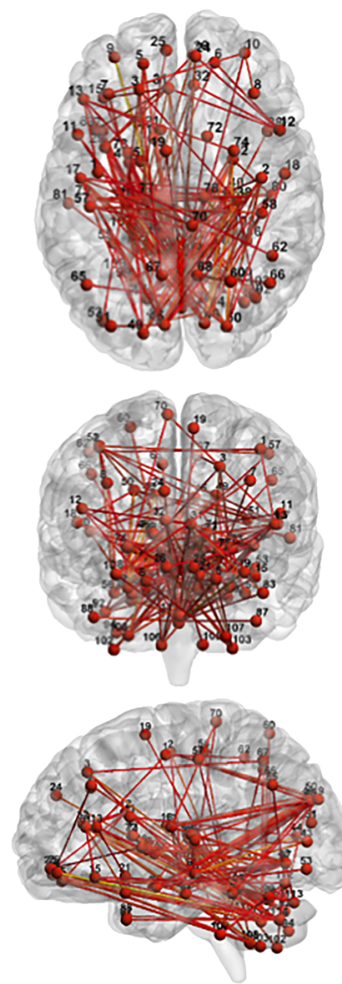

b

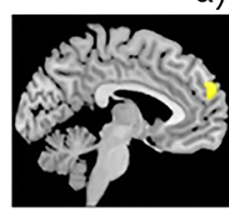

a) DMN

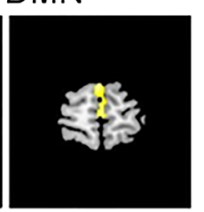

$\underbrace{}_{\text {t-value }}$

Fig. 3 Increased Functional Connectivity (FC) in Relapsing-Remitting Multiple Sclerosis (MS) patients correlated with information processing efficiency at whole brain connectome (A, using primary thresholds of $\mathrm{p}<$

of subcortical regions, namely the thalamus, putamen, caudate and pallidum and visual and sensorimotor FC networks was also highly influenced by the WM lesion load. These subnetworks have been related to clinical factors and the most commonly reported and often debilitating symptom in MS, i.e. fatigue (Brownlee et al. 2017; Finke et al. 2015). Reduced cerebellar FC with increased lesion load has also previously been reported in MS, and was inversely correlated with global clinical disability (Dogonowski et al. 2014). Finally, a higher lesion load, which leads to decreased FC in the DMN, salience and attention networks (DAN and VAN) is likely to affect $\mathrm{p}<0.001$
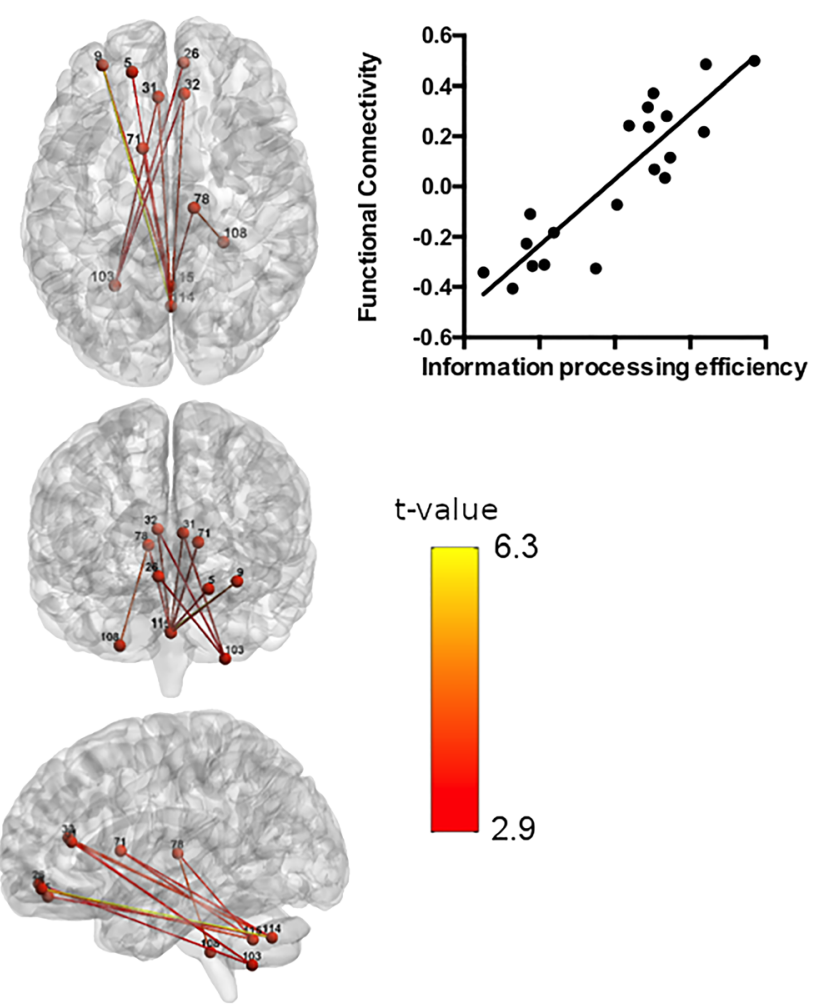

t-value

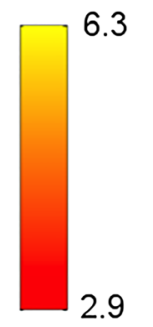

c) $S M N$

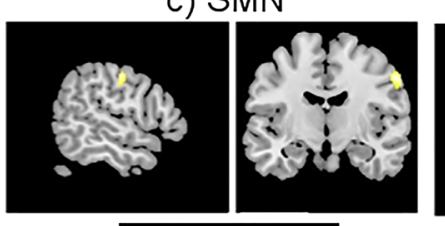

d) VAN

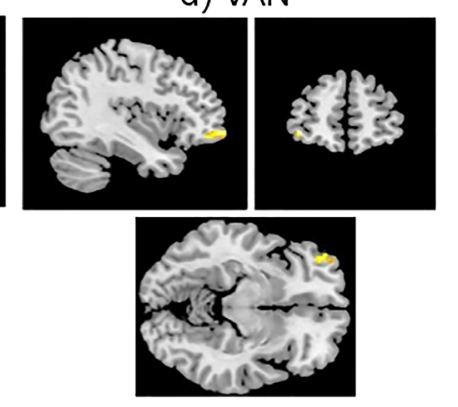

$0.01, \mathrm{p}<0.005$ and $\mathrm{p}<0.001$, corrected for multiple comparisons) and within Resting State Networks (RSNs, corrected for multiple comparisons) (B)

cognitive and information processing efficiency in MS (Louapre et al. 2014; Rocca et al. 2017). .

\section{Association between information processing efficiency and FC in RRMS}

In accordance and as previously mentioned, $\mathrm{FC}$ alterations in MS have frequently been associated with impairment of cognitive function (Hawellek et al. 2011; Louapre et al. 2014; Rocca et al. 2016). Herein, we have observed a positive correlation between connectome's FC and information 
Table 4 Correlation between information processing efficiency composite and RSNs FC in RRMS (multiple regressions, corrected for multiple comparisons, $\mathrm{p}<0.05$ )

\begin{tabular}{llllr}
\hline Correlation & Network & Regions & Peak MNI coordinates & $\begin{array}{c}\text { Cluster size } \\
\text { Score }\end{array}$ \\
\hline Positive & DMN & Right superior medial Frontal gyrus & $0,54,30$ & 151 \\
& & Left superior medial Frontal gyrus & $-2,62,30$ & 3.11 \\
& Precuneus & Left Angular gyrus & $-40,-68,50$ & 3.03 \\
& Sensorimotor & Right Postcentral gyrus & $56,-10,42$ & 3.91 \\
& VAN & Left middle Orbitofrontal cortex & $-38,54,-6$ & 61 \\
& & Left inferior Orbitofrontal cortex & $-42,46,-10$ & 3.69 \\
& & & & 2.69 \\
\hline
\end{tabular}

processing efficiency for both short and long-range connections, with a higher prominence for long-range connections, especially between frontal and cerebellar regions. In particular, impaired FC in the long-range pathway between frontal and cerebellar brain regions is reported to contribute to failure of cognitive compensation in MS (Bonnet et al. 2010), as subnetworks involving these regions have been positively correlated with the cognitive efficiency (Dogonowski et al. 2014; Rocca et al. 2017; Rocca et al. 2016). Specifically, when considering the RSNs only on the RRMS group, we observed that $\mathrm{FC}$ of the frontal regions of the DMN and VAN displayed a positive correlation with information processing efficiency. In fact DMN FC is considered to be an important component of cognition and is proposed to be essential for efficient cognitive performance (Newton et al. 2011). This observation is consistent with findings showing that cognitively impaired patients with MS typically display a decreased DMN deactivation during task performance (Rocca et al. 2014), and decreased FC during resting state (Louapre et al. 2014). Abnormal FC of the VAN and precuneus networks was also found in MS patients with cognitive impairment (Petracca et al. 2017). Finally our results also highlight the role of the sensorimotor network's FC as a possible surrogate for MS (Castellazzi et al. 2018; Rocca et al. 2017). Overall, both cognitive and sensoriomotor RSNs are likely to be influenced by disease progression and appear to be complementary in determining FC alterations, as reduced $\mathrm{FC}$ in cognitive and increased $\mathrm{FC}$ in sensorimotor RSNs appear to differentiate MS with short from longer disease duration (Castellazzi et al. 2018).

\section{Limitations and conclusions}

This study presents limitations that should be addressed. Firstly it is a cross-sectional design with a limited sample size of RRMS patients. Additionally, we cannot exclude that factors such as diffuse microscopic WM abnormalities and gray matter lesions might also contribute to the relationship between brain FC and clinical dysfunction. We also recognize the importance of using a T2 sequence for lesion volume calculation, the lesion location, its individual volume and perform lesion filling, and note that this study does not address that variables. Therefore, considering additional radiological evidence (e.g. different image acquisitions, atrophy, etc), as well as other clinical, disability and disease duration, together with exploring anatomical templates more recent than AAL (which presents sub-par deep grey matter segmentation), should be addressed by future studies. Finally, results from our exploratory approach cannot infer the role of FC alterations in compensatory or neural disruption hypothesis, which warrants a longitudinal and multimodal approach. Nonetheless, to the best of our knowledge there are no prior reports which have sought to evaluate both the whole brain connectome and RSNs FC on the same study and cohort of MS patients, the influence of WM lesions and information processing efficiency.

It is hoped that our results may contribute to the clarification of the literature inconsistency regarding FC alterations with MS patients, as well as to better delineate the impact of WM lesioning in the FC between key network hubs as the frontal regions, thalamus, cerebellum and the DMN, and its relation to information processing efficiency.

Acknowledgments We are thankful to all study participants. We thank Edward Ganz for the revision of the writing and thank the collaboration of Dr. José Grilo Gonçalves and Dr. Filipe Palavra (that helped with participant's recruitment at Hospital dos Covões in Coimbra).

Funding information This study was conducted at the Psychology Research Centre (PSI/01662), School of Psychology, University of Minho, and supported by the Portuguese Foundation for Science and Technology and the Portuguese Ministry of Science, Technology and Higher Education (UID/PSI/01662/2019), through the national funds (PIDDAC) and PIC/IC/83290/2007.

\section{Compliance with ethical standards}

Conflict of interest The authors declare that the research was conducted in the absence of any commercial or financial relationships that could be construed as a potential conflict of interest. 
Ethical approval This study was conducted in accordance with the principles expressed in the Declaration of Helsinki and approved by the Ethics Committee of Hospital Geral de Coimbra (Covões) - Centro Hospitalar de Coimbra and from the Ethical Board of the School of Psychology (University of Minho).

Informed consent The study goals and tests were explained to the participants and informed consent was obtained from all individual participants included in the study.

\section{References}

Beck, A. T., Ward, C. H., Mendelson, M., Mock, J., \& Erbaugh, J. (1961). An inventory for measuring depression. Archives of General Psychiatry, 4, 561-571.

Beckmann, C. F., \& Smith, S. M. (2004). Probabilistic independent component analysis for functional magnetic resonance imaging. IEEE Transactions on Medical Imaging, 23(2), 137-152. https://doi.org/ 10.1109/tmi.2003.822821.

Benedict, R. H., Cookfair, D., Gavett, R., Gunther, M., Munschauer, F., Garg, N., \& Weinstock-Guttman, B. (2006). Validity of the minimal assessment of cognitive function in multiple sclerosis (MACFIMS). Journal of the International Neuropsychological Society, 12(4), 549-558.

Benedict, R. H., Fischer, J. S., Archibald, C. J., Arnett, P. A., Beatty, W. W., Bobholz, J., Chelune, G. J., Fisk, J. D., Langdon, D. W., Caruso, L., Foley, F., LaRocca, N., Vowels, L., Weinstein, A., DeLuca, J., Rao, S. M., \& Munschauer, F. (2002). Minimal neuropsychological assessment of MS patients: A consensus approach. The Clinical Neuropsychologist, 16(3), 381-397. https://doi.org/10.1076/clin. 16.3.381.13859.

Benedict, R. H., Smerbeck, A., Parikh, R., Rodgers, J., Cadavid, D., \& Erlanger, D. (2012). Reliability and equivalence of alternate forms for the symbol digit modalities test: Implications for multiple sclerosis clinical trials. Multiple Sclerosis, 18(9), 1320-1325. https://doi. org/10.1177/1352458511435717.

Bonavita, S., Gallo, A., Sacco, R., Corte, M. D., Bisecco, A., Docimo, R., Lavorgna, L., Corbo, D., Costanzo, A. D., Tortora, F., Cirillo, M., Esposito, F., \& Tedeschi, G. (2011). Distributed changes in defaultmode resting-state connectivity in multiple sclerosis. Multiple Sclerosis, 17(4), 411-422. https://doi.org/10.1177/ 1352458510394609 .

Bonnet, M. C., Allard, M., Dilharreguy, B., Deloire, M., Petry, K. G., \& Brochet, B. (2010). Cognitive compensation failure in multiple sclerosis. Neurology, 75(14), 1241-1248. https://doi.org/10.1212/WNL. 0b013e3181f612e3.

Braley, T. J., \& Chervin, R. D. (2010). Fatigue in multiple sclerosis: Mechanisms, evaluation, and treatment. Sleep, 33(8), 1061-1067.

Brownlee, W. J., Hardy, T. A., Fazekas, F., \& Miller, D. H. (2017). Diagnosis of multiple sclerosis: Progress and challenges. Lancet, 389(10076), 1336-1346. https://doi.org/10.1016/S0140-6736(16) 30959-X.

Calabrese, M., Magliozzi, R., Ciccarelli, O., Geurts, J. J., Reynolds, R., \& Martin, R. (2015). Exploring the origins of grey matter damage in multiple sclerosis. Nature Reviews. Neuroscience, 16(3), 147-158. https://doi.org/10.1038/nrn3900.

Cardinal, K. S., Wilson, S. M., Giesser, B. S., Drain, A. E., \& Sicotte, N. L. (2008). A longitudinal fMRI study of the paced auditory serial addition task. Multiple Sclerosis, 14(4), 465-471. https://doi.org/10. $1177 / 1352458507084263$.

Castellazzi, G., Debernard, L., Melzer, T. R., Dalrymple-Alford, J. C., D'Angelo, E., Miller, D. H., Gandini Wheeler-Kingshott, C. A. M., \& Mason, D. F. (2018). Functional connectivity alterations reveal complex mechanisms based on clinical and radiological status in mild relapsing remitting multiple sclerosis. Frontiers in Neurology, 9, 690-690. https://doi.org/10.3389/fneur.2018.00690.

Cavanna, A. E., \& Trimble, M. R. (2006). The precuneus: A review of its functional anatomy and behavioural correlates. Brain, 129(Pt 3), 564-583. https://doi.org/10.1093/brain/awl004.

Chiaravalloti, N. D., \& DeLuca, J. (2008). Cognitive impairment in multiple sclerosis. Lancet Neurology, 7(12), 1139-1151. https://doi.org/ 10.1016/S1474-4422(08)70259-X.

Christodoulou, C., Krupp, L. B., Liang, Z., Huang, W., Melville, P., Roque, C., Scherl, W. F., Morgan, T., MacAllister, W., Li, L., Tudorica, L. A., Li, X., Roche, P., \& Peyster, R. (2003). Cognitive performance and MR markers of cerebral injury in cognitively impaired MS patients. Neurology, 60(11), 1793-1798.

De Santis, S., Granberg, T., Ouellette, R., Treaba, C. A., Herranz, E., Fan, Q., et al. (2019). Evidence of early microstructural white matter abnormalities in multiple sclerosis from multi-shell diffusion MRI. Neuroimage Clin, 22, 101699. https://doi.org/10.1016/j.nicl.2019. 101699.

Deco, G., \& Kringelbach, M. L. (2014). Great expectations: Using wholebrain computational Connectomics for understanding neuropsychiatric disorders. Neuron, 84(5), 892-905. https://doi.org/10.1016/j. neuron.2014.08.034

DeLuca, J., Chelune, G. J., Tulsky, D. S., Lengenfelder, J., \& Chiaravalloti, N. D. (2004). Is speed of processing or working memory the primary information processing deficit in multiple sclerosis? Journal of Clinical and Experimental Neuropsychology, 26(4), 550 562. https://doi.org/10.1080/13803390490496641.

Dogonowski, A. M., Andersen, K. W., Madsen, K. H., Sorensen, P. S., Paulson, O. B., Blinkenberg, M., \& Siebner, H. R. (2014). Multiple sclerosis impairs regional functional connectivity in the cerebellum. Neuroimage Clin, 4, 130-138. https://doi.org/10.1016/j.nicl.2013. 11.005 .

Droby, A., Yuen, K. S., Muthuraman, M., Reitz, S. C., Fleischer, V., Klein, J., Gracien, R. M., Ziemann, U., Deichmann, R., Zipp, F., \& Groppa, S. (2016). Changes in brain functional connectivity patterns are driven by an individual lesion in MS: A resting-state fMRI study. Brain Imaging and Behavior, 10(4), 1117-1126. https://doi. org/10.1007/s11682-015-9476-3.

Emanuele, P., Maria, A. R., Paola, V., Gianna, C. R., Claudio, G., Giancarlo, C., et al. (2017). Gray matter trophism, cognitive impairment, and depression in patients with multiple sclerosis. Multiple Sclerosis Journal, 23(14), 1864-1874. https://doi.org/10.1177/ 1352458517692886.

Filippi, M., Bar-Or, A., Piehl, F., Preziosa, P., Solari, A., Vukusic, S., \& Rocca, M. A. (2018). Multiple sclerosis. Nature Reviews Disease Primers, 4(1), 43. https://doi.org/10.1038/s41572-018-0041-4.

Filippi, M., Rocca, M. A., De Stefano, N., Enzinger, C., Fisher, E., Horsfield, M. A., et al. (2011). Magnetic resonance techniques in multiple sclerosis: The present and the future. Archives of Neurology, 68(12), 1514-1520. https://doi.org/10.1001/archneurol. 2011.914.

Finke, C., Schlichting, J., Papazoglou, S., Scheel, M., Freing, A., Soemmer, C., Pech, L. M., Pajkert, A., Pfüller, C., Wuerfel, J. T., Ploner, C. J., Paul, F., \& Brandt, A. U. (2015). Altered basal ganglia functional connectivity in multiple sclerosis patients with fatigue. Multiple Sclerosis, 21(7), 925-934. https://doi.org/10.1177/ 1352458514555784.

Fleischer, V., Radetz, A., Ciolac, D., Muthuraman, M., GonzalezEscamilla, G., Zipp, F., \& Groppa, S. (2019). Graph theoretical framework of brain networks in multiple sclerosis: A review of concepts. Neuroscience, 403, 35-53. https://doi.org/10.1016/j. neuroscience.2017.10.033.

Fox, M. D., \& Raichle, M. E. (2007). Spontaneous fluctuations in brain activity observed with functional magnetic resonance imaging. 
Nature Reviews. Neuroscience, 8(9), 700-711. https://doi.org/10. 1038/nrn2201.

Hawellek, D. J., Hipp, J. F., Lewis, C. M., Corbetta, M., \& Engel, A. K. (2011). Increased functional connectivity indicates the severity of cognitive impairment in multiple sclerosis. Proceedings of the National Academy of Sciences of the United States of America, 108(47), 19066-19071. https://doi.org/10.1073/pnas.1110024108.

He, Y., Dagher, A., Chen, Z., Charil, A., Zijdenbos, A., Worsley, K., \& Evans, A. (2009). Impaired small-world efficiency in structural cortical networks in multiple sclerosis associated with white matter lesion load. Brain, 132(Pt 12), 3366-3379. https://doi.org/10.1093/ brain/awp089.

Jaeger, S., Paul, F., Scheel, M., Brandt, A., Heine, J., Pach, D., Witt, C. M., Bellmann-Strobl, J., \& Finke, C. (2018). Multiple sclerosisrelated fatigue: Altered resting-state functional connectivity of the ventral striatum and dorsolateral prefrontal cortex. Multiple Sclerosis Journal, 25(4), 554-564. https://doi.org/10.1177/ 1352458518758911.

Jongen, P. J., Ter Horst, A. T., \& Brands, A. M. (2012). Cognitive impairment in multiple sclerosis. Minerva Medica, 103(2), 73-96.

Langen, C. D., Zonneveld, H. I., White, T., Huizinga, W., Cremers, L. G. M., de Groot, M., Ikram, M. A., Niessen, W. J., \& Vernooij, M. W. (2017). White matter lesions relate to tract-specific reductions in functional connectivity. Neurobiology of Aging, 51, 97-103. https://doi.org/10.1016/j.neurobiolaging.2016.12.004.

Liu, Y., Dai, Z., Duan, Y., Huang, J., Ren, Z., Liu, Z., Dong, H., Shu, N., Vrenken, H., Wattjes, M. P., Barkhof, F., He, Y., \& Li, K. (2016). Whole brain functional connectivity in clinically isolated syndrome without conventional brain MRI lesions. European Radiology, 26(9), 2982-2991. https://doi.org/10.1007/s00330-015-4147-8.

Liu, Y., Duan, Y., Dong, H., Barkhof, F., Li, K., \& Shu, N. (2018). Disrupted module efficiency of structural and functional brain Connectomes in clinically isolated syndrome and multiple sclerosis. Frontiers in Human Neuroscience, 12, 138. https://doi.org/10.3389/ fnhum.2018.00138.

Louapre, C., Perlbarg, V., Garcia-Lorenzo, D., Urbanski, M., Benali, H., Assouad, R., et al. (2014). Brain networks disconnection in early multiple sclerosis cognitive deficits: An anatomofunctional study. Human Brain Mapping, 35(9), 4706-4717. https://doi.org/10. 1002/hbm.22505.

Magliozzi, R., Reynolds, R., \& Calabrese, M. (2018). MRI of cortical lesions and its use in studying their role in MS pathogenesis and disease course. Brain Pathology, 28(5), 735-742. https://doi.org/10. 1111/bpa.12642.

Marques, P., Moreira, P., Magalhaes, R., Costa, P., Santos, N., Zihl, J., et al. (2016). The functional connectome of cognitive reserve. Human Brain Mapping. https://doi.org/10.1002/hbm.23242.

Minagar, A., Barnett, M. H., Benedict, R. H., Pelletier, D., Pirko, I., Sahraian, M. A., Frohman, E., \& Zivadinov, R. (2013). The thalamus and multiple sclerosis: Modern views on pathologic, imaging, and clinical aspects. Neurology, 80(2), 210-219. https://doi.org/10. 1212/WNL.0b013e31827b910b.

Newton, A. T., Morgan, V. L., Rogers, B. P., \& Gore, J. C. (2011). Modulation of steady state functional connectivity in the default mode and working memory networks by cognitive load. Human Brain Mapping, 32(10), 1649-1659. https://doi.org/10.1002/hbm. 21138.

Patel, K. R., Tobyne, S., Porter, D., Bireley, J. D., Smith, V., \& Klawiter, E. (2018). Structural disconnection is responsible for increased functional connectivity in multiple sclerosis. Brain Structure and Function, 223(5), 2519-2526. https://doi.org/10.1007/s00429-0181619-z.

Patrick, E., Christodoulou, C., Krupp, L. B., \& New York State, M. S. C. (2009). Longitudinal correlates of fatigue in multiple sclerosis. Multiple Sclerosis, 15(2), 258-261. https://doi.org/10.1177/ 1352458508097466.
Petracca, M., Saiote, C., Bender, H. A., Arias, F., Farrell, C., Magioncalda, P., Martino, M., Miller, A., Northoff, G., Lublin, F., \& Inglese, M. (2017). Synchronization and variability imbalance underlie cognitive impairment in primary-progressive multiple sclerosis. Scientific Reports, 7, 46411. https://doi.org/10.1038/ srep46411.

Polman, C. H., Reingold, S. C., Banwell, B., Clanet, M., Cohen, J. A., Filippi, M., Fujihara, K., Havrdova, E., Hutchinson, M., Kappos, L., Lublin, F. D., Montalban, X., O'Connor, P., Sandberg-Wollheim, M., Thompson, A. J., Waubant, E., Weinshenker, B., \& Wolinsky, J. S. (2011). Diagnostic criteria for multiple sclerosis: 2010 revisions to the McDonald criteria. Annals of Neurology, 69(2), 292-302. https:// doi.org/10.1002/ana.22366.

Rocca, M. A., Valsasina, P., Absinta, M., Riccitelli, G., Rodegher, M. E., Misci, P., Rossi, P., Falini, A., Comi, G., \& Filippi, M. (2010). Default-mode network dysfunction and cognitive impairment in progressive MS. Neurology, 74(16), 1252-1259. https://doi.org/10. 1212/WNL.0b013e3181d9ed91.

Rocca, M. A., Valsasina, P., Hulst, H. E., Abdel-Aziz, K., Enzinger, C., Gallo, A., . . . Group, M. f. S. (2014). Functional correlates of cognitive dysfunction in multiple sclerosis: A multicenter fMRI study. Human Brain Mapping, 35(12), 5799-5814. doi:https://doi. org/10.1002/hbm.22586.

Rocca, M. A., Valsasina, P., Leavitt, V. M., Rodegher, M., Radaelli, M., Riccitelli, G. C., et al. (2017). Functional network connectivity abnormalities in multiple sclerosis: Correlations with disability and cognitive impairment. Multiple Sclerosis, 1352458517699875. https://doi.org/10.1177/1352458517699875.

Rocca, M. A., Valsasina, P., Meani, A., Falini, A., Comi, G., \& Filippi, M. (2016). Impaired functional integration in multiple sclerosis: A graph theory study. Brain Structure \& Function, 221(1), 115-131. https://doi.org/10.1007/s00429-014-0896-4.

Roosendaal, S. D., Schoonheim, M. M., Hulst, H. E., Sanz-Arigita, E. J., Smith, S. M., Geurts, J. J., \& Barkhof, F. (2010). Resting state networks change in clinically isolated syndrome. Brain, 133(Pt 6), 1612-1621. https://doi.org/10.1093/brain/awq058.

Sbardella, E., Petsas, N., Tona, F., \& Pantano, P. (2015a). Resting-State fMRI in MS: General concepts and brief overview of its application. BioMed Research International, 2015, 212693. https://doi.org/10. 1155/2015/212693.

Sbardella, E., Tona, F., Petsas, N., Upadhyay, N., Piattella, M., Filippini, N., Prosperini, L., Pozzilli, C., \& Pantano, P. (2015b). Functional connectivity changes and their relationship with clinical disability and white matter integrity in patients with relapsing-remitting multiple sclerosis. Multiple Sclerosis Journal, 21(13), 1681-1692. https://doi.org/10.1177/1352458514568826.

Schoonheim, M. M., Meijer, K. A., \& Geurts, J. J. (2015). Network collapse and cognitive impairment in multiple sclerosis. Frontiers in Neurology, 6, 82. https://doi.org/10.3389/fneur.2015.00082.

Shirer, W. R., Ryali, S., Rykhlevskaia, E., Menon, V., \& Greicius, M. D. (2012). Decoding subject-driven cognitive states with whole-brain connectivity patterns. Cerebral Cortex, 22(1), 158-165. https://doi. org/10.1093/cercor/bhr099.

Skokou, M., Soubasi, E., \& Gourzis, P. (2012). Depression in multiple sclerosis: A review of assessment and treatment approaches in adult and pediatric populations. ISRN Neurology, 2012, 427102. https:// doi.org/10.5402/2012/427102.

Soares, J. M., Magalhaes, R., Moreira, P. S., Sousa, A., Ganz, E., Sampaio, A., et al. (2016). A Hitchhiker's guide to functional magnetic resonance imaging. Frontiers in Neuroscience, 10, 515. https:// doi.org/10.3389/fnins.2016.00515.

Strauss, E., Sherman, E. M. S., \& Spreen, O. (2007). Compendium of neuropsychological tests: Administration, norms, and commentary. Applied Neuropsychology, 14(1), 62-63. https://doi.org/10.1080/ 09084280701280502. 
Sumowski, J. F., Chiaravalloti, N., Wylie, G., \& Deluca, J. (2009). Cognitive reserve moderates the negative effect of brain atrophy on cognitive efficiency in multiple sclerosis. Journal of the International Neuropsychological Society, 15(4), 606-612. https:// doi.org/10.1017/s1355617709090912.

Tahed1, M., Levine, S. M., Greenlee, M. W., Weissert, R., \& Schwarzbach, J. V. (2018). Functional connectivity in multiple sclerosis: Recent findings and future directions. Frontiers in Neurology, 9, 828. https://doi.org/10.3389/fneur.2018.00828.

Tewarie, P., Schoonheim, M., \& Hillebrand, A. (2018a). Structural and functional neuroimaging in multiple sclerosis: From atrophy, lesions to global network disruption. In C. Habas (Ed.), The neuroimaging of brain diseases: Structural and functional advances (pp. 171213). Cham: Springer International Publishing.

Tewarie, P., Steenwijk, M. D., Brookes, M. J., Uitdehaag, B. M. J., Geurts, J. J. G., Stam, C. J., \& Schoonheim, M. M. (2018b). Explaining the heterogeneity of functional connectivity findings in multiple sclerosis: An empirically informed modeling study. Human Brain Mapping, 39(6), 2541-2548. https://doi.org/10.1002/hbm. 24020.

Tzourio-Mazoyer, N., Landeau, B., Papathanassiou, D., Crivello, F., Etard, O., Delcroix, N., Mazoyer, B., \& Joliot, M. (2002).
Automated anatomical labeling of activations in SPM using a macroscopic anatomical parcellation of the MNI MRI single-subject brain. NeuroImage, 15(1), 273-289. https://doi.org/10.1006/nimg. 2001.0978.

van den Heuvel, M. P., \& Hulshoff Pol, H. E. (2010). Exploring the brain network: A review on resting-state fMRI functional connectivity. European Neuropsychopharmacology, 20(8), 519-534. https://doi. org/10.1016/j.euroneuro.2010.03.008.

Vaz Serra, A. S., Abreu, J. P. (1973). Aferição dos Quadros Clínicos Depressivos - Ensaio de Aplicação do «Inventário Depressivo de Beck» a uma Amostra Portuguesa de Doentes Deprimidos. Separata da Coimbra Médica, XX(VI), 623-644.

Zalesky, A., Fornito, A., \& Bullmore, E. T. (2010). Network-based statistic: Identifying differences in brain networks. NeuroImage, 53(4), 1197-1207. https://doi.org/10.1016/j.neuroimage.2010.06.041.

Zhang, D., \& Raichle, M. E. (2010). Disease and the brain's dark energy. Nature Reviews. Neurology, 6(1), 15-28. https://doi.org/10.1038/ nrneurol.2009.198.

Publisher's note Springer Nature remains neutral with regard to jurisdictional claims in published maps and institutional affiliations. 